\title{
Potent Antitumor T-Cell Memory Is Generated by Curative Viral Oncolytic Immunotherapy But Not Curative Chemotherapy
}

\author{
YANHUA GAO ${ }^{1}$ and IRA BERGMAN $N^{1,2,3}$ \\ Departments of ${ }^{1}$ Pediatrics, ${ }^{2}$ Neurology, and ${ }^{3}$ Immunology, University of Pittsburgh School of Medicine, \\ Children's Hospital of Pittsburgh, Pittsburgh, PA, U.S.A.
}

\begin{abstract}
Background: Late developing breast cancer metastases are common and lethal despite treatment with adjuvant chemotherapy at the time of primary tumor excision. Stimulation of an antitumor immune response is an alternative strategy for preventing this devastating development. Materials and Methods: A mouse model of the human epidermal growth factor receptor 2 (HER2/neu)-positive mammary cancer was used to compare the antitumor memory $T$-cell response following tumor cure by viral oncolytic immunotherapy, chemotherapy, surgical excision, or surgical excision plus virus infection. Memory T-cell response was assessed by functional in vivo assays. Results: Antitumor T-cell memory was generated most powerfully by curative viral oncolytic immunotherapy and poorly by curative chemotherapy. Cure by surgical excision generated an immune antitumor response which was increased by neo-adjuvant virus infection. CD4 memory T-cells were most potent. Conclusion: Virus infection of tumor generates an antitumor memory immune response and chemotherapy suppresses this response. Clinical trials testing adjuvant immune stimulation instead of chemotherapy may be worth exploring because memory antitumor T-cells have the unique potential to find and eliminate small nests of metastatic cancer cells in sanctuary sites and prevent emergence of tumors from dormant cancer cells.
\end{abstract}

Late developing breast cancer metastases are common and lethal despite treatment with adjuvant and neo-adjuvant

This article is freely accessible online.

Correspondence to: Ira Bergman and Yanhua Gao, Children's Hospital of Pittsburgh of UPMC, One Children's Hospital Drive, 4401 Penn Avenue, Pittsburgh, PA 15224, U.S.A. Tel: +1 4126927176, e-mail: ira.bergman@chp.edu; yanhua.gao@chp.edu

Key Words: Memory T-cells, immunotherapy, oncotherapy, vesicular stomatitis virus, chemotherapy. chemotherapy at the time of primary tumor resection. About 40,890 women and 440 men were projected to die of breast cancer in 2016 in the United States and 522,000 people died globally of breast cancer in 2012. About $90 \%$ of the deaths are due to metastatic disease to sites such as lung, liver, bone and brain. Somewhat less than half of these patients presented with metastatic disease but the others succumbed to only late developing metastases. Even many with metastatic disease at presentation had treatment which produced initial remission but succumbed later to recurrent metastases. Currently there is no treatment to permanently eradicate metastases and median survival with metastatic breast cancer is 3 years. Despite various treatments, there has been no improvement in this severe mortality in the last 20 years $(1,2)$.

The use of tumor-targeting T-cells is a new approach that has had stunning successes when adapted as chimeric antigen receptor T-cells (CAR T-cells) in inhibiting and eliminating some types of hematological cancer (3-7). This therapy is not yet effective against solid tumors and is very expensive because the T-cells are manufactured separately for each individual patient. We previously showed in an experimental mouse model of solid tumors that viral oncolytic immunotherapy with a targeted replicating recombinant vesicular stomatitis virus (rrVSV) generated highly potent therapeutic antitumor memory T-cells that matured from 30 to 100 days after initial antigen exposure and were resistant to inhibition by suppressor cells (8-12). Long established experiments, however, have shown that curative surgical excision of implanted mouse tumors also generated immunity that prevented growth of the same re-implanted tumor (13, 14). The current work aimed to determine whether tumor cure by different treatments resulted in different strengths of antitumor T-cell memory. Tumors were implanted in either the peritoneum or the mammary gland. Treatments included surgical excision, excision plus virus infection, viral oncolytic immunotherapy and chemotherapy. Memory T-cell response was assessed by functional in vivo assays. The ultimate goal is to be able to use treatments clinically that generate 
maximum antitumor immune responses which then prevent outgrowth of tumor metastases.

\section{Materials and Methods}

Cells, antibodies, chemicals and animals. D2F2/E2, a mouse mammary tumor line that was stably transfected with a vector expressing the human epidermal growth factor receptor 2 (HER2/neu) gene and its parent cell line, D2F2, were a generous gift from Dr. Wei-Zen Wei, (Karmanos Cancer Institute, Wayne State University, Detroit, MI, USA) in 2001. Early passage cells were frozen and periodically thawed for experimental use or restocking. Thawed cells were used experimentally for 5-10 passages. Mycoplasma testing was negative using the Impact III PCR profile from IDEXX (RADIL, Columbia, MO, USA). Monoclonal antibody to cytotoxic T-lymphocyte-associated protein 4 (CTLA4) (9H10) was obtained commercially (BioXcell Fermentation/Purification Services, West Lebanon, NH, USA). Mice were 8 to 20 weeks of age and weighed $20-25 \mathrm{~g}$. Thy $1.2 \mathrm{BALB} / \mathrm{c}$ mice were obtained from Taconic (Hudson, NY, USA). A mating pair of Thy $1.1 \mathrm{BALB} / \mathrm{c}$ mice were purchased from the Jackson Laboratory (Strain name: CBy.PL (B6)-Thy $1^{a} / \mathrm{ScrJ}$ ) and bred on site. Animal studies were approved by the institutional Animal Research and Care Committee Protocol \# IS00005591.

Creation of rrVSV. A replicating virus expressing the following properties was created from vector components as previously described (15): Preferential infection of cells expressing human HER2/neu, expression of mouse granulocyte-macrophage colonystimulating factor, and expression of enhanced green fluorescent protein. Construction used vectors generously supplied by Dr. John K. Rose (Department of Pathology, Yale University, New Haven, CT, USA), Dr. Irvin S. Y. Chen (Department of Microbiology, Immunology, Molecular Genetics, University of California, Los Angeles, CA, USA) and Genentech Inc., South San Francisco, CA, USA).

Tumor implants: Peritoneal, subcutaneous and cisterna magna $(C M)$. Female BALB/c Thy 1.2 mice were implanted intraperitoneally (i.p.) with $2 \times 10^{6} \mathrm{D} 2 \mathrm{~F} 2 / \mathrm{E} 2$ cells in $300 \mu \mathrm{l}$ phosphate buffered saline (PBS). Subcutaneous injections in the mammary gland (16) were performed by injection of $5 \times 10^{5}$ D2F2/E2 cells in $50 \mu \mathrm{l}$ PBS within the subcutaneous space of the mammary gland. $\mathrm{CM}$ injections were performed as previously described by injecting $2 \times 10^{5} \mathrm{D} 2 \mathrm{~F} 2 / \mathrm{E} 2$ cells in $20 \mu \mathrm{l}$ PBS into the cerebrospinal fluid immediately inferior to the occipital bone of the skull (10).

Tumor curative treatments: Viral immunotherapy, chemotherapy, surgical excision, intratumoral rrVSV. Viral immunotherapy consisted of treatment on day 3 after peritoneal implant with i.p. rrVSV, $1 \times 10^{8} \mathrm{ID}$, on day 4 with $200 \mu \mathrm{g}$ anti-CTLA4 i.p. and on day 5 with $\sim 100 \mathrm{mg} / \mathrm{kg}$ cyclophosphamide $i . p$. Chemotherapy consisted of treatment on day 3 after peritoneal implant with i.p. $3.2 \mathrm{mg} / \mathrm{kg}$ doxorubicin and $60 \mathrm{mg} / \mathrm{kg}$ gemcitabine followed by weekly injections of the same chemotherapy, twice more. Surgical excision of subcutaneous tumors was performed under isoflurane anesthesia on day 15 after implant. Intratumoral virus on day 9 after implant consisted of $2 \times 10^{7}$ ID rrVSV injected into 1-3 locations depending on the size of the tumor. Cure was determined by survival for more than 90 days after tumor implant. The number of animals in each group is given in the Figures.

Cell collection. Memory cells were obtained from spleens or peritoneal cells of cured animals. Animals were sacrificed prior to cell harvesting. Spleens were harvested, minced and ground through a $70 \mu \mathrm{M}$ nylon cell strainer (BD Falcon, Franklin Lakes, NJ, USA). Red blood cells were removed using RBC lysis buffer (Alfa Aesar Haverhill, MA, USA). Peritoneal washings were performed by injecting $10 \mathrm{ml}$ of sterile PBS into the peritoneum through a 16-gauge needle which was left in place. Five minutes later, all the fluid that it was possible to aspirate easily into a syringe was collected; usually $9.0 \mathrm{ml}$ was collected. All cells were washed twice with PBS and re-suspended in PBS. Total T-cells, $\mathrm{CD}^{+}{ }^{+} \mathrm{T}$-cells and $\mathrm{CD} 8{ }^{+} \mathrm{T}$-cells were isolated by positive or negative selection using an autoMACS ${ }^{\mathrm{TM}}$ separator and the appropriate antibody microbeads according to the manufacturer's instructions (Miltenyi Biotec, Auburn, CA, USA): CD90 (Thy1.2), CD4 (L3T4), CD8a (Ly-2). Spleen cells were labelled with carboxyfluorescein succinimidyl ester (CFSE) (Vybrant CFDA SE Cell Tracer Kit; Life Technologies Corp. Carlsbad, CA, USA) following the manufacturer's instructions.

Adoptive therapy. Peritoneal tumors were established in host animals and treated 3 days later by adoptive transfer of splenocytes or isolated $\mathrm{CD}^{+}{ }^{+}$or $\mathrm{CD}^{+} \mathrm{T}$-cells from animals cured by different means. Total splenocytes from each mouse were divided into aliquots containing $90 \%$ or $10 \%$ of the total cells. Different recipients received one of these aliquots.

As previously described, host animals were pre-treated with cyclophosphamide 1 day before the transfer of memory cells (8). Animals were assessed three times per week for ascites, abdominal nodules and signs of poor health such as low activity, poor grooming, rough coat, hunched posture and dehydration, and sacrificed if they developed ascites, nodules or any of these signs.

In one set of experiments, CFSE-labeled spleen cells were transferred from donor Thy 1.2 mice to peritoneal tumor-bearing host Thy 1.1 animals. Host animals were sacrificed 5 days later and the number and proliferation of Thy $1.2 \mathrm{CD}^{+}$and $\mathrm{CD} 8^{+} \mathrm{T}$-cells in the peritoneum was identified by Thy $1.2, \mathrm{CD}^{+}, \mathrm{CD}^{+}$, tetramer and CFSE staining using flow cytometry.

Flow cytometry. A total of $1 \times 10^{6}$ peritoneal cells were suspended in ice-cold PBS $/ 0.1 \%$ bovine serum albumin $/ 0.2 \%$ azide and stained with combinations of the following antibodies: CD4allophycocyanin (APC)-eFluor 780 (eBioscience, San Diego, CA, USA), CD8a-phycoerythrin (PE)-cyanine7 (eBioscience), CD90.2PE (Becton Dickinson, Mountainview, CA, USA), Live-Dead fixable red Kit (Thermo Fisher Scientific, Pittsburgh, PA, USA) and H-2K (d)/TYLPTNASL, human HER2 p63 tetramer conjugated with Alexa 647 (NIH Tetramer Core Facility at Emory University, Atlanta, GA, USA). Immunofluorescence was quantified using an LSR Fortesa (Becton Dickinson, Mountainview, CA, USA).

Statistical analysis. The log-rank statistic was used to compare survival among the treatment groups. An unpaired two-tailed $t$-test was used for all other statistical comparisons with similar variance assumed across independent groups. Data are presented as means \pm SEM. PRISM software was used to analyze the data (GraphPad Software, Inc., La Jolla, CA, USA). 
A

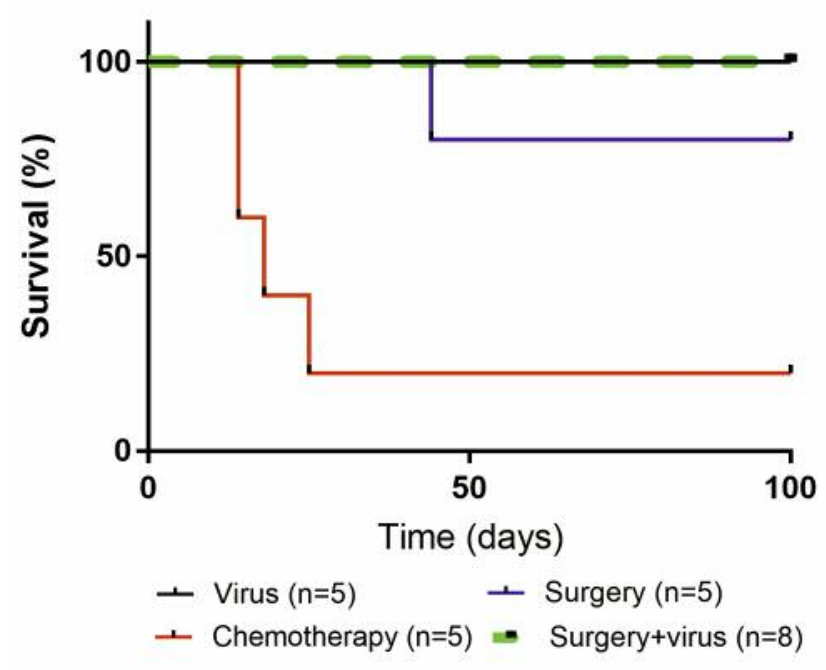

B

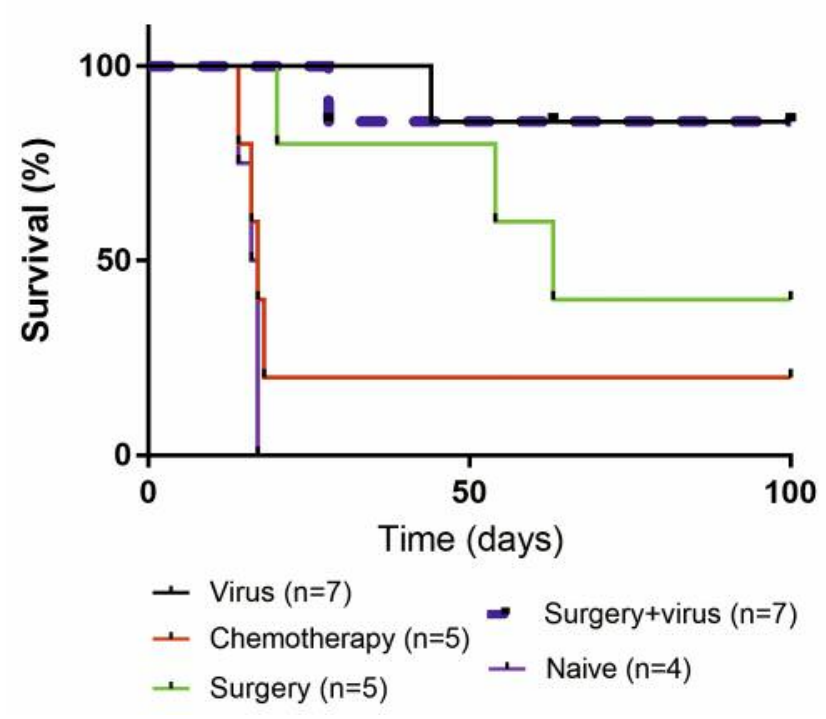

\section{Results}

Cure by viral oncolytic immunotherapy prevents reimplantation; cure by chemotherapy does not. Implanted tumors at two sites were cured by one of four treatments: rrVSV oncolytic viral immunotherapy; chemotherapy; surgical excision; surgical excision plus neo-adjuvant intra-tumoral rrVSV infection.

Cured animals were challenged $>90$ days later with the same cell type either in the peritoneum, the subcutaneous space or the CM of the brain. Cure of i.p. tumors with viral oncolytic immunotherapy prevented re-implantation at all sites overall, $96 \%$ of the time (Figure 1). In contrast, cure of
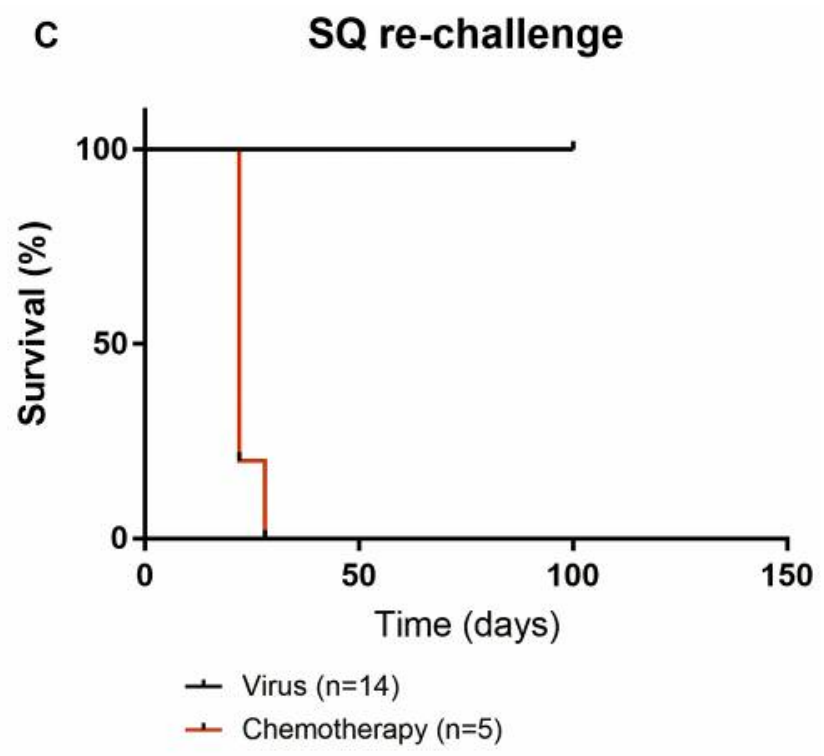

Figure 1. Survival following tumor re-challenge in peritoneum $(A)$, cisterna magna $(C M)(B)$ or subcutaneous tissue $(S Q)(C)$ in animals previously cured as follows: Virus: Intraperitoneal tumor cured with replicating recombinant vesicular stomatitis virus (rrVSV) oncolytic viral immunotherapy. Chemotherapy: Intraperitoneal tumor cured with chemotherapy. Surgery: subcutaneous tumors cured by surgical excision. Surgery+virus: Subcutaneous tumors cured by surgical excision plus neoadjuvant intra-tumoral rrVSV infection. On peritoneal re-challenge, survival time was significantly better in animals cured with viral immunotherapy than with chemotherapy ( $p=0.0128$; log-rank test). On CM re-challenge, survival time was significantly better in animals cured with viral immunotherapy than with chemotherapy $(p=0.0089)$. On $S Q$ re-challenge, survival time was significantly better in in animals cured with viral immunotherapy than with chemotherapy $(p<0.0001)$.

i.p. tumors with chemotherapy prevented reimplantation $20 \%$ of the time in the peritoneum or the CM and not at all in the subcutaneous space $(0 \%)$ (Figure 1). Mice with subcutaneous tumors cured with excision plus virus were challenged i.p. and $\mathrm{CM}$ and resisted re-implantation $93.3 \%$ of the time (Figure 1A and B). Mice with subcutaneous tumors cured with excision alone were challenged i.p. and CM and resisted re-implantation $60 \%$ of the time (Figure $1 \mathrm{~A}$ and $\mathrm{B}$ ). As detailed in Figure 1, the difference in resistance to reimplantation between cure with viral oncolytic immunotherapy and cure with chemotherapy was statistically significant at each of the three challenge sites. There was no statistically significant difference in resistance to re- 
challenge between cure with surgical excision alone and excision plus viral infection.

Cure by viral oncolytic immunotherapy generates memory T-cells that can be passively transferred to cure established peritoneal tumors in host animals; cure by chemotherapy does not. Donor animals consisted of four groups of tumor cures as above. Host animals with established 3-day peritoneal tumors were treated with spleen cells from a single donor animal. We previously demonstrated that in this experimental model, T-cells are the active therapeutic cells in the spleen (12). In one set of experiments, host mice received $90 \%$ of the total complement of spleen cells from the donor and in a second set of experiments, host mice received $10 \%$ of the total complement of spleen cells from the donor. As shown in Figure 2A for 90\% transfers, cure was $100 \%$ when donors were cured with viral oncolytic immunotherapy or surgical excision plus neo-adjuvant virus, $80 \%$ when donors were cured by surgical excision alone and $0 \%$ when donors were cured with chemotherapy. There was a statistically significant difference in outcome between chemotherapy donors and each of the other donors ( $p=0.0015$ compared with viral oncolytic immunotherapy; $p=0.01$ compared with surgical excision alone; $p=0.0015$ compared with surgical excision plus intratumor virus). No cures were achieved from any donors when $10 \%$ spleen cells were transferred but survival time was significant better with donor cells from animals cured with viral oncolytic immunotherapy $(p=0.007)$ or with surgical excision plus intratumor virus $(p=0.0021)$ than with chemotherapy. Survival time was also significantly better with donors cured with surgical excision plus intratumor virus than donors cured with surgical excision alone $(p=0.0018)$ (log-rank test for all comparisons).

CD4 memory T-cells are responsible for cure with adoptive transfer and are more effective from donors cured with surgical excision plus intratumoral virus than surgical excision alone. A third set of transfer experiments compared the therapeutic efficacy of $\mathrm{CD} 4^{+}$and $\mathrm{CD} 8^{+} \mathrm{T}$-cells alone. $\mathrm{CD} 8^{+} \mathrm{T}$-cells alone did not produce any cures (Figure 3 ). $\mathrm{CD} 4^{+}$T-cells alone from animals cured with either viral oncolytic immunotherapy or surgical excision plus intratumor virus cured $66.6 \%$ of hosts whereas cells from donors cured with surgical excision alone cured only $16.6 \%$ (Figure $3 ; p=0.02$ comparing surgical excision plus intratumor virus with excision alone).

The specific antitumor memory $\mathrm{CD} 8^{+}$and $C D 4^{+}$T-cell response is greater in animals cured with viral oncolytic immunotherapy or surgical excision plus intratumoral virus than those cured with chemotherapy. The specific antitumor memory $\mathrm{CD}^{+}{ }^{+} \mathrm{T}$-cell response in the peritoneum was quantified by flow cytometry analyses using a tetramer displaying the immunodominant p63 epitope of the HER2/neu receptor protein. Cured mice were challenged once with i.p. tumor and sacrificed 5 days later with harvest of peritoneal lavage cells. The response to tumor challenge was $\sim 100$-fold higher in animals cured with viral oncolytic immunotherapy than those cured with chemotherapy alone (Figure $4 ; p=0.05$ ). The number of tetramer-positive cells was $\sim 5$-fold higher in animals cured with surgical excision plus intratumor virus than those cured with surgical excision alone (Figure 4; $p=0.027$ ) and $~ 20$-fold higher than those cured with chemotherapy (Figure $4 ; p=0.01$ ). The variability of results was large probably because some animals responded to a different class I epitope than p63 and these produced a much lower number of p63 tetramer-positive $\mathrm{CD} 8^{+} \mathrm{T}$-cells than the animals responding to the usual p63 immunodominant epitope.

The antitumor memory $\mathrm{CD} 4^{+}$response was identified using congenic animals because we did not have a tumorspecific class II tetramer. CFSE-labeled spleen cells were transferred from donor cured Thy 1.2 animals into host Thy 1.1 mice that were implanted i.p. with the same tumor cells. Peritoneal cells were harvested by lavage 5 days later and donor memory $\mathrm{CD} 4{ }^{+} \mathrm{T}$-cells that migrated to the tumor in the peritoneum were identified by Thy 1.2 staining and CFSE-low replication. Donor memory CD $8^{+}$ T-cells were identified by Thy 1.2 and tetramer staining. The number of replicating CFSE-low donor $\mathrm{CD} 4^{+} \mathrm{T}$-cells was significantly greater from animals cured with viral oncolytic immunotherapy $(p=0.01)$ and surgical excision plus intratumoral virus ( $p=0.02$; unpaired two-tailed $t$-test) than animals cured with chemotherapy indicating that the former cures generated a more powerful CD4 response than chemotherapy (Figure 5A). The number of donor tetramer-positive T-cells in this transfer experiment (Figure 5B) was strikingly similar to the number obtained following challenge in the cured animals themselves (Figure 4) and once again showed a remarkably small number of 748 tetramer-positive T-cells from donors cured with chemotherapy compared with 70,288 from mice cured with viral oncolytic immunotherapy and 206,365 from mice cure by surgical excision plus intratumoral virus (Figure 5B). The number from animals cured with surgery alone was also low, 3993. The only statistically significant difference in this tetramer data was the higher number from donors cured with viral oncolytic immunotherapy compared to those cured with chemotherapy $(p=0.02$; unpaired two-tailed $t$-test). The other large differences did not reach statistical significance because of the unavoidable large variability when relying on results from a single tetramer, as noted above. Illustrative flow cytometry graphs from individual animals are shown in Figure 5C. 
A Survival following transfer: $90 \%$ spleen

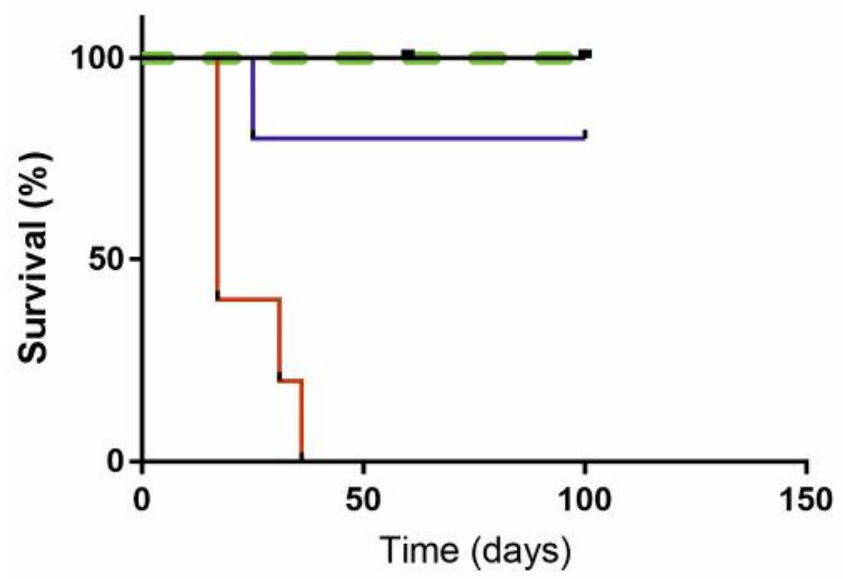

$\begin{array}{ll}\perp \text { Virus }(n=6) & \perp \text { Surgery }(n=5) \\ \perp \text { Chemotherapy }(n=5) & - \text { Surgery+virus }(n=5)\end{array}$
B Survival following transfer: $10 \%$ spleen

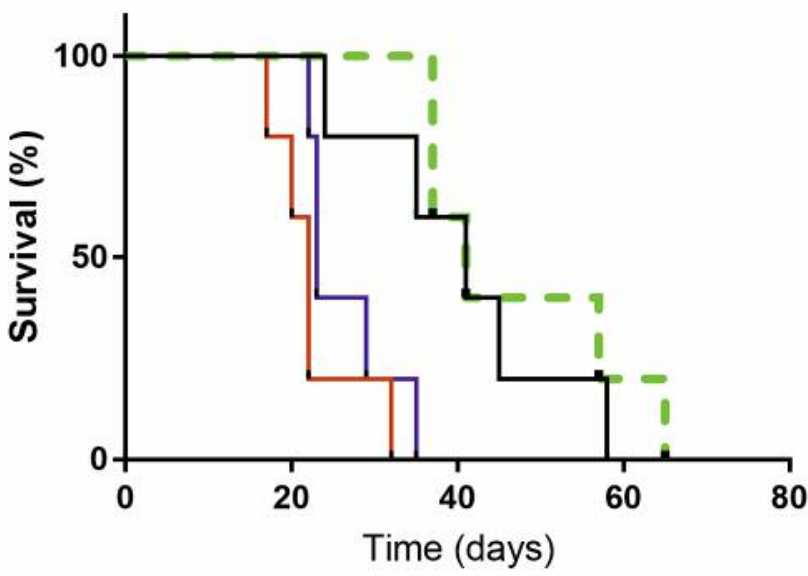

$\begin{array}{ll}\perp \text { Virus }(n=5) & \perp \text { Surgery }(n=5) \\ \perp \text { Chemotherapy }(n=5) & \rightarrow \text { Surgery+virus }(n=5)\end{array}$

Figure 2. Survival of host animals with established peritoneal tumors following transfer of spleen cells from previously cured donors: A: Transfer of $90 \%$ spleen from a single donor. B: Transfer of 10\% spleen from a single donor. Survival time was significantly worse with chemotherapy than each of the other cured animals in those receiving $90 \%$ transfer ( $p=0.0015$ compared with viral immunotherapy; $p=0.01$ compared with surgical excision alone; $p=0.0015$ compared with surgery plus virus; log-rank test). Survival time was significantly better in animals receiving $10 \%$ transfer cured with viral immunotherapy than chemotherapy $(p=0.0089)$ and with surgery plus virus than surgical excision alone $(p=0.0018)$.

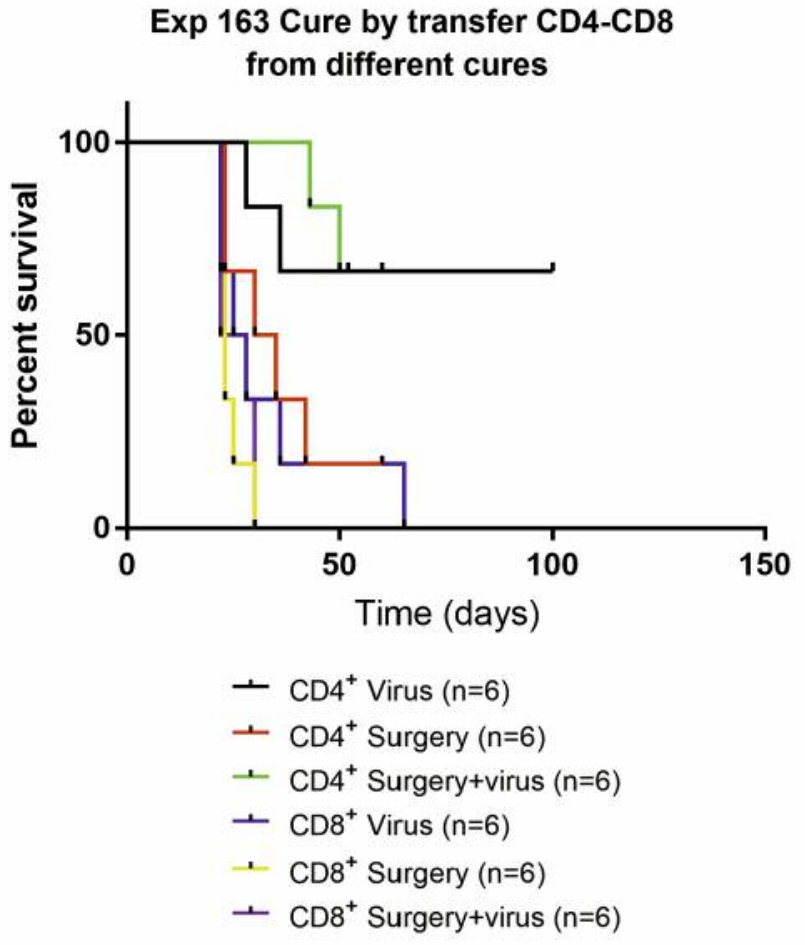

Figure 3. Survival of host animals with established peritoneal tumors following transfer of $\mathrm{CD}^{+}$or $\mathrm{CD} 8^{+}$T-cells from previously cured donors. Survival time was significantly better following transfer of memory $\mathrm{CD}^{+} \mathrm{T}$-cells from animals cured with surgical excision plus virus than surgical excision alone ( $p=0.02$; log-rank test).

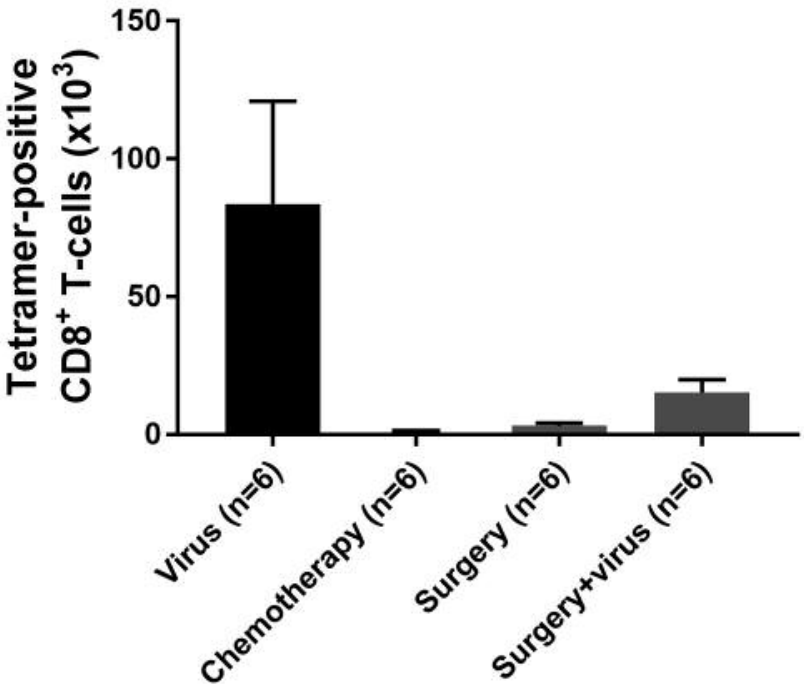

Figure 4. Tumor-specific CD8 ${ }^{+}$T-cell response in the peritoneum to peritoneal tumor challenge in previously cured animals. The tetramer response was significantly higher in animals cured with viral immunotherapy than chemotherapy $(p=0.05)$ and significantly higher in those cured with surgical excision plus intratumoral virus than those without ( $p=0.027$; unpaired two-sided $t$-test). Data are mean $\pm S E M$. 
Subcutaneous tumors treated with intratumoral virus generate acute effectors that mediate concomitant immunity. As demonstrated above, animals cured of subcutaneous tumors by surgical excision alone generated potent antitumor T-cell immunity, but several experiments showed that more powerful immunity occurred when excision was preceded by neo-adjuvant intratumor rrVSV injection. Further evidence for immune boosting by virus infection was obtained by implanting i.p. tumor in animals with established 8-day subcutaneous tumors. The experimental group received intratumor rrVSV on day 9 and the control group received media. All subcutaneous tumors were surgically excised on day 15 . Survival studies showed that all animals cured by excision alone succumbed to the i.p. tumors, whereas $50 \%$ of the animals cured by excision plus neo-adjuvant rrVSV survived (Figure 6; $p=0.0015$; log-rank test).

\section{Discussion}

The major finding in this study is that rrVSV promotes a potent memory antitumor immune response following acute curative therapy and chemotherapy inhibits this memory immune response. The clinical implications are large because outgrowth of metastases months and years after complete surgical eradication of primary breast cancer are a leading cause of mortality in this disease. This study suggests that adjuvant and neo-adjuvant chemotherapy used to eradicate and suppress active metastases may have the deleterious effect of suppressing the antitumor immune response to late growing metastases. rrVSV infection of tumor either therapeutically or as a neo-adjuvant immunization combined with surgical excision may generate enough antitumor T-cells to prevent late outgrowth of metastases. These clinical correlations are advanced tentatively because the model system used in this study has significant differences from the natural human disease. The tumors are implanted and not slowly growing over many years. The treatments yield full cure whereas clinically, dormant metastatic cells remain present after surgical excision of primary tumors. Nevertheless, the conclusion is clear that rrVSV infection of tumor promotes antitumor memory and chemotherapy inhibits it. In this study, cure by chemotherapy rarely prevented tumor growth following re-challenge at any site tested including peritoneal, CM and subcutaneous. Peritoneal challenge resulted in an extraordinary low number of responding $\mathrm{CD}^{+}$or $\mathrm{CD}^{+}{ }^{+}$T-cells. Donor memory T-cells from these animals did not suppress tumor growth in host animals with implanted peritoneal tumors. In contrast, cure by viral oncolytic immunotherapy prevented tumor growth following re-challenge at every site. Peritoneal challenge resulted in high numbers of responding $\mathrm{CD}^{+}$or $\mathrm{CD} 4^{+} \mathrm{T}$-cells. High numbers of donor memory T-cells from these animals cured established peritoneal tumors in host animals and low numbers suppressed them.
The results comparing the antitumor memory immune response following cure by surgical excision with and without neo-adjuvant rrVSV infection were subtler. On every measure, the results showed a higher memory immune response following surgical excision with rrVSV infection than without infection, but a high baseline response following cure with surgical excision alone meant that statistical significance was achieved only for some measures: survival following transfer of low-dose memory T-cells, survival following transfer of memory $\mathrm{CD}^{+}{ }^{+} \mathrm{T}$-cells and the number of tetramer-positive $\mathrm{CD}^{+}$T-cells following peritoneal re-challenge. Added evidence for the antitumor immune-stimulating effect of neoadjuvant rrVSV infection was provided in experiments showing acute concomitant immunity generated by surgical excision with rrVSV infection but not without. A similar effect using a different virus was recently reported (17). Overall, the consistent positive effect of neo-adjuvant rrVSV infection across many measures in an animal model with many unavoidable variabilities supports its importance in improving antitumor immunity.

This study did not determine the mechanisms of the differential response, but confirmed our previous work in this tumor model that memory $\mathrm{CD}^{+} \mathrm{T}$-cells were the key instruments of tumor cure $(8,12) . \mathrm{CD}^{+} \mathrm{T}$-cells can kill cancer cells directly themselves, or indirectly by stimulating and recruiting $\mathrm{CD}^{+} \mathrm{T}$-cells and various other immune cells to the tumor (18). Viral oncolytic immunotherapy and surgical excision plus neo-adjuvant virus infection of tumor generated more antitumor memory $\mathrm{CD} 4^{+} \mathrm{T}$-cells than other treatments. Future work must determine whether more memory $\mathrm{CD}^{+}{ }^{+} \mathrm{T}$-cells or different kinds of memory $\mathrm{CD} 4^{+}$ T-cells were generated and the basic molecular and cellular mechanisms of their generation. An advantage of these experiments is that they were performed in mice with a normal diversity of T-cells and not transgenic mice with monoclonal T-cells. The findings were supported by functional in vivo assays, which are direct measures that

Figure 5. Migration of tumor-specific T-cells to peritoneal tumor in hosts following transfer of spleen cells from previously cured donors. A: Tumor-specific memory $\mathrm{CD}^{+} \mathrm{T}$-cells in the peritoneum identified by Thy 1.2 staining and carboxyfluorescein succinimidyl ester (CFSE) replication. The number was significantly greater from donors cured with viral oncolytic immunotherapy $(p=0.01)$ and surgical excision plus intratumoral virus $(p=0.02)$ than donors cured with chemotherapy. B: Tumor-specific memory CD8+T-cells in the peritoneum identified by Thy 1.2 and tetramer staining. The number was significantly greater from donors cured with viral oncolytic immunotherapy than donors cured with chemotherapy $(p=0.02)$. C: Representative flow cytometric data from peritoneal cells selected for Thy 1.2 staining. As shown, almost all tetramer-positive T-cells were CFSE-low, indicating active replication. Data are the mean \pm SEM; unpaired two-tailed t-test. 

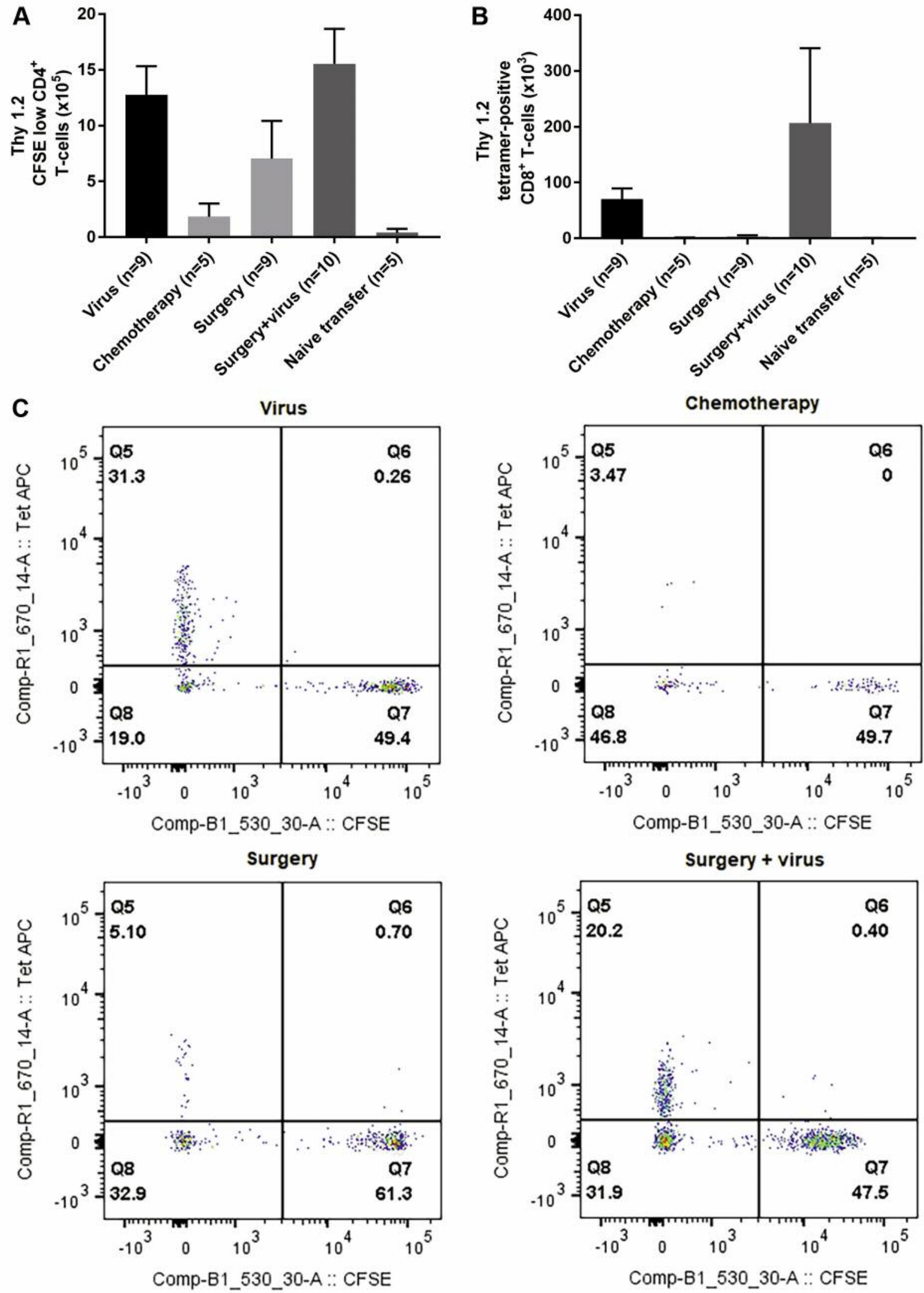

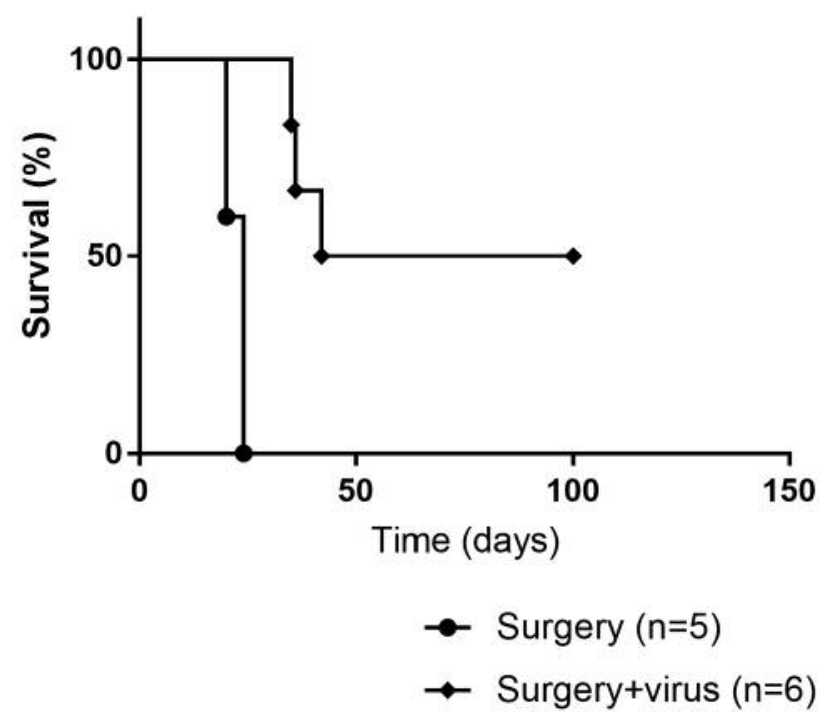

Figure 6. Survival of mice following peritoneal challenge prior to surgical excision of subcutaneous tumors. Survival was significantly improved, including $50 \%$ survival, in animals who received replicating recombinant vesicular stomatitis virus infection of subcutaneous tumors prior to excision $(p=0.0015$; log-rank test).

integrate all the interactions occurring in the animals, as opposed to in vitro cytokine or surface marker assays, which may give variable results when directly compared and may not correlate with clinical immunity $(19,20)$. Memory Tcells were defined by their presence 90 days after tumor cure and not by surface markers which can be imprecise, especially in mice $(21,22)$.

This work is viewed as one step in developing a vaccine that generates therapeutic antitumor memory T-cells. These T-cells would be permanently available to survey the body and eliminate recurrence of tumor at the primary site or spread from dormant metastatic sites at a very early stage just as viral memory T-cells do for herpes simplex virus (HSV), Varicella zoster virus (VZV), Epstein-Barr virus (EBV) and Papovavirus. Early small tumors have not developed strong mechanisms to resist immune attack and these mechanisms are less effective against memory T-cells than acute effector T-cells $(9,23)$. Although all successful vaccinations to date have been antibody-mediated and no T-cell-dependent vaccinations, with the possible exception of bacille CalmetteGuerin for disseminated tuberculosis, have yet been developed to prevent infectious diseases or tumor recurrence $(24,25)$, this study demonstrates that effective antitumor memory $\mathrm{CD}^{+} \mathrm{T}$-cells can be generated by viral oncotherapy. The next step will be to test the use of viral oncotherapy in combination with other immune stimulants to generate therapeutic antitumor memory T-cells in the continuous presence of tumor antigen as occurs when active, dormant or latent metastases are present after primary tumor resection. Vaccination in the presence of chronic clinical infections such as tuberculosis, malaria and human immunodeficiency virus and in a mouse model system of lymphocytic choriomeningitis virus have not been successful $(26,27)$. On the other hand, effective anti-virus memory CD8 T-cells are generated after natural infection with HSV, VZV, EBV and Papovavirus despite the persistence of latent virus.

This study clearly shows that therapeutic chemotherapy can severely inhibit the development of antitumor T-cell memory. It suggests that adjuvant chemotherapy following primary breast cancer resection may worsen the outcome for patients with dormant metastases at risk for developing late recurrence. On the other hand, adjuvant chemotherapy may be essential for patients with active metastases at risk for early recurrence. Clinical studies are required to determine whether current diagnostic tools can distinguish these states and whether each requires a different adjuvant therapy at the time of initial tumor resection. The results will be relevant to many types of cancer with late metastases, including breast, kidney, prostate and malignant melanoma.

\section{Funding}

US Army Medical Research Breast Cancer IDEA Award (BC101672) to IB.

\section{Conflicts of Interest}

IB: US Patent Application 7,429,481 entitled "Targeting viruses using a modified Sindbis glycoprotein" was granted September 30, 2008. The targeted virus is tested in this animal work. IB has no affiliation with any company. YG has no conflict of interest to report.

\section{Acknowledgements}

The Authors thank Drs. Wei-Zen Wei, John K. Rose, Irvin S. Y. Chen and Genentech Inc. who very generously supplied materials as noted in the text. We thank Joshua Michel, Senior Technician and Flow Core Manager for invaluable technical assistance with Flow Cytometry.

The Authors gratefully acknowledge the assistance of the NIH Tetramer Core Facility at Emory University and Dale Long, facility manager, for generously supplying all the tetramer reagents. The NIH Tetramer Facility is supported by contract HHSN272 201300006C from the National Institute of Allergy and Infectious Diseases, a component of the National Institutes of Health in the Department of Health and Human Services.

\section{References}

1 Department of Defense Breast Cancer Research: The Breast Cancer Landscape. 2016.

2 Kennecke H, Yerushalmi R, Woods R, Cheang MCU, Voduc D, Speers CH, Nielsen TO and Gelmon K: Metastatic behavior of breast cancer subtypes. J Clin Oncol 28(20): 3271-3277, 2010. 
3 Roncarolo MG and Battaglia M: Regulatory t-cell immunotherapy for tolerance to self antigens and alloantigens in humans. Nat Rev Immunol 7(8): 585-598, 2007.

4 McLachlan SM and Rapoport B: Autoimmune hypothyroidism: T-Cells caught in the act. Nat Med 10(9): 895-896, 2004.

5 Bingaman AW and Farber DL: Memory t cells in transplantation: Generation, function, and potential role in rejection. Am J Transplant 4(6): 846-852, 2004.

6 Davila ML and Sadelain M: Biology and clinical application of CAR T-cells for B-cell malignancies. Int J Hematol 104(1): 617, 2016.

7 Degn $\mathrm{M}$ and Kornum BR: Type 1 narcolepsy: A CD8 (+) T-cellmediated disease? Ann N Y Acad Sci 1351: 80-88, 2015.

8 Gao Y, Whitaker-Dowling P, Griffin JA and Bergman I: Treatment with targeted vesicular stomatitis virus generates therapeutic multifunctional anti-tumor memory CD4 T-cells. Cancer Gene Ther 19(4): 282-291, 2012.

9 Gao Y, Whitaker-Dowling P and Bergman I: Memory antitumor T-cells resist inhibition by immune suppressor cells. Anticancer Res 35(9): 4593-4597, 2015.

10 Gao Y, Whitaker-Dowling P, Barmada MA, Basse PH and Bergman I: Viral infection of implanted meningeal tumors induces antitumor memory T-cells to travel to the brain and eliminate established tumors. Neuro Oncol 17(4): 536-544, 2015.

11 Wentworth L, Meyers JV, Alam S, Russ AJ, Suresh M and Cho CS: Memory T-cells are uniquely resistant to melanoma-induced suppression. Cancer Immunol Immunother 62(1): 149-159, 2013

12 Gao Y, Barmada MA and Bergman I: Antitumor memory T-cells become functionally mature from 30 to 100 days in a mouse model of neoplasia. Anticancer Res 38(1): 147-157, 2018.

13 Prehn RT and Main JM: Immunity to methylcholanthreneinduced sarcomas. J Natl Cancer Inst 18(6): 769-778, 1957.

14 Wahl DV, Chapman WH, Hellstrom I and Hellstrom KE: Transplantation immunity to individually unique antigens of chemically induced bladder tumors in mice. Int J Cancer 14(1): 114-121, 1974.

15 Bergman I, Whitaker-Dowling $\mathrm{P}$, Gao $\mathrm{Y}$ and Griffin JA: Preferential targeting of vesicular stomatitis virus to breast cancer cells. Virology 330(1): 24-33, 2004.

16 Pulaski BA and Ostrand-Rosenberg S: Mouse 4t 1 breast tumor model. Curr Protoc Immunol Chapter 20: Unit 20.22, 2001.
17 Bourgeois-Daigneault MC, Roy DG, Aitken AS, El Sayes N, Martin NT, Varette O, Falls T, St-Germain LE, Pelin A, Lichty BD, Stojdl DF, Ungerechts G, Diallo JS and Bell JC: Neoadjuvant oncolytic virotherapy before surgery sensitizes triple-negative breast cancer to immune checkpoint therapy. Sci Transl Med 10(422): 03, 2018.

18 Brown DM: Cytolytic CD4 cells: Direct mediators in infectious disease and malignancy. Cell Immunol 262(2): 89-95, 2010.

19 Macatangay BJC, Zheng L, Rinaldo CR, Landay AL, Pollard RB, Pahwa S, Lederman MM and Bucy RP: Comparison of immunologic assays for detecting immune responses in HIV immunotherapeutic studies: Aids clinical trials group trial a5181. Clin Vaccine Immunol 17(9): 1452-1459, 2010.

20 Masucci GV, Cesano A, Hawtin R, Janetzki S, Zhang J, Kirsch I, Dobbin KK, Alvarez J, Robbins PB, Selvan SR, Streicher HZ, Butterfield LH and Thurin M: Validation of biomarkers to predict response to immunotherapy in cancer: Volume I - Pre-analytical and analytical validation. J Immunother Cancer 4(1): 76, 2016.

21 Jameson SC and Masopust D: Understanding subset diversity in T-cell memory. Immunity 48(2): 214-226, 2018.

22 Steinert EM, Schenkel JM, Fraser KA, Beura LK, Manlove LS, Igyarto BZ, Southern PJ and Masopust D: Quantifying memory CD8 T-cells reveals regionalization of immunosurveillance. Cell 161 (4): 737-749, 2015.

23 McNeel DG: Therapeutic cancer vaccines: How much closer are we? Biodrugs 32(1): 1-7, 2018.

24 Panagioti E, Klenerman P, Lee LN, van der Burg SH and Arens R: Features of effective T-cell-inducing vaccines against chronic viral infections. Front Immunol 9(276): 276, 2018.

25 Gilbert SC: T-Cell-inducing vaccines - what's the future. Immunology 135(1): 19-26, 2012.

26 Seder RA and Hill AV: Vaccines against intracellular infections requiring cellular immunity. Nature 406(6797): 793-798, 2000.

27 Wherry EJ, Blattman JN, Murali-Krishna K, van der Most R and Ahmed R: Viral persistence alters CD8 T-cell immunodominance and tissue distribution and results in distinct stages of functional impairment. J Virol 77(8): 4911-4927, 2003.

Received October 11, 2018

Revised October 29, 2018

Accepted November 1, 2018 